\title{
Towards the description of water adsorption in slit-like nanochannels with grafted molecular brushes. Density functional theory
}

\author{
V.M. Trejos ${ }^{1}$, M. Aguilar ${ }^{2}$, S. Sokołowski ${ }^{3}$, O. Pizid $\left.4\right|^{k}$ \\ ${ }^{1}$ Instituto de Ciencias Básicas e Ingeniería, Universidad Autónoma del Estado de Hidalgo, Carretera \\ Pachuca-Tulancingo Km. 4.5, Col. Carboneras, C.P. 42184, Mineral de la Reforma, Hidalgo, México \\ 2 Instituto de Química, Universidad Nacional Autónoma de México, Circuito Exterior, 04510, Cd. de México, \\ México \\ 3 Department for the Modelling of Physico-Chemical Processes, Maria Curie-Skłodowska University, \\ Lublin 20-031, Poland \\ ${ }^{4}$ Instituto de Química, Universidad Nacional Autónoma de México, Circuito Exterior, 04510, Cd. de México, \\ México
}

Received January 19, 2020, in final form March 10, 2020

\begin{abstract}
We have explored a model for adsorption of water into slit-like nanochannels with two walls chemically modified by grafted polymer layers forming brushes. A version of density functional method is used as theoretical tools. The water-like fluid model adopted from the work of Clark et al. [Mol. Phys., 2006, 104, 3561] adequately reproduces the bulk vapour-liquid coexistence envelope. The polymer layer consists of chain molecules in the framework of pearl-necklace model. Each chain molecule is chemically bonded to the pore walls by a single terminating segment. Our principal focus is in the study of the dependence of polymer layer height on grafting density and in the microscopic structure of the interface between adsorbed fluid and brushes. Thermal response of these properties upon adsorption is investigated in detail. The results are of importance to understand shrinking and swelling of the molecular brushes in the nanochannels.
\end{abstract}

Key words: associating fluids, density functional theory, adsorption, molecular brushes, water model

\section{Introduction}

This manuscript has been prepared as a tribute to Prof. Ihor Mryglod, distinguished Ukrainian scientist in the field of statistical physics, on behalf of his 60th birthday. Dr. Mryglod has made several important contributions along different lines of research within the theory of liquids. Some of his works related to our scientific interests are focused on the comprehension of the equilibrium and out-of-equilibrium behaviour of water and other liquids as well as on the relation between the collective variables method and the density functional approaches in the theory of inhomogeneous fluids, see e.g., [1]-3].

Synthesis and design of smart interfaces is one of the important areas of applied research and a challenging subject for theoretical investigations. Grafted polymer brushes represent an essential ingredient of this type of materials. Design of adaptive surfaces requires the use of malleable polymers whose properties change in response to environmental stimuli such as temperature, electric field, $\mathrm{pH}$ or light [4-13]. Profound comprehension of surface/interface properties is a prerequisite of developing rational synthetic strategies and new structures with functionalities for novel applications [6, 7, 12, 13].

Conformational behaviour of grafted polymer layers is determined by the length of chain molecules, grafting density, and the curvature of the supporting substrate. Usually, three regimes are distinguished

*E-mail: oapizio@gmail.com 
in the brush formation, namely, mushroom type structure, crossover regime and highly stretched regime. Specifically, at low grafting density and with poor solvent, the polymer chains prefer to be collapsed. With an increasing grafting density, a semi-dilute polymer brush regime is realized, the configurational entropy of chains becomes reduced compared to mushroom-like structures. At high grafting densities, polymer chains become stretched due to an increased mutual confinement that creates a rather concentrated polymer brush layer [10, 14].

From theoretical perspective, computer simulation methods, see e.g., [15- -23$]$ and [24] for a quite recent review, and entirely theoretical approaches have been applied to describe systems involving tethered brushes. The latter include the self-consistent field approaches [25-29], and density functional (DF) theories [30]. The DF approaches represent versatile tools to describe an ample variety of brush-fluid models and have been applied in the studies of lipids, co-polymers, grafted polymers, polymer/colloid, and polymer/nanoparticle systems [31-35]. In particular, Yu and $\mathrm{Wu}$ [36, 37] developed a successful version of the DF theory to describe systems of nonuniform polymers.

In this work, we present a continuation of the project, see [38-42], focused on the study of the behaviour of water in complex porous media. At the first stage, in [38], we studied thermodynamic properties of a set of water-like models designed in [43] by applying the method similar to [44]. The principal idea behind the modelling of [43] is to reproduce the liquid-vapour (LV) coexistence of water using square-well attraction and site-site chemical association without resorting to electrostatic inter-particle interactions. We incorporated this model into a DF approach to describe the behaviour of water-like models in slit-like pores [39]. Our theoretical findings appeared to be in agreement with computer simulation results of much more sophisticated water models in pores [45-47]. The most recent part of our studies along this line of research is focused on the effects of chemical modification of slit-like pore walls by grafted polymer layers [40-42]. Specifically, we studied the adsorption of water in slit-like pores with grafted polymer layers on the walls resorting to a version of the DF theory. Shrinking or swelling of molecular brushes induced by phase transitions in confined water [42] was explored.

In the present contribution we continue the investigation of the model from [40-42] concerned with the properties of water in pores with chemically modified walls. However, the principal purpose of this work is to describe the thermal response of grafted polymer layers to the conditions of adsorption. In contrast to [40-42], we assume that there is an attractive interaction between segments of grafted chain molecules. Therefore, the brushes are thermal on their own, not only due to the action of the adsorbed fluid. This modelling is closer to the experimental setup [6] and permits to obtain additional insights into the behaviour of this class of adsorption systems.

The manuscript is organized as follows. In sections 2 and 3 the setup is described as briefly as possible, because a detailed description of the model and theory has been given elsewhere [42]. In addition, a few essential equations are given in the supplementary material for the sake of convenience of the reader. In section 4, the original results are discussed. Next, the conclusions and summary are given.

\section{Model}

We consider the fluid model as one-component fluid of associating molecules. Each fluid molecule has four associative sites designated by A, B, C and D, inscribed into a spherical core [48, 50]. The set of all the sites is denoted by $\Gamma$. The pair inter-molecular potential between molecules 1 and 2 depends on the center-to-center distance and orientations,

$$
u(12)=u_{\mathrm{ff}}\left(r_{12}\right)+\sum_{\alpha \in \Gamma} \sum_{\beta \in \Gamma} u_{\alpha \beta}\left(\mathbf{r}_{\alpha \beta}\right),
$$

where $\mathbf{r}_{\alpha \beta}=\mathbf{r}_{12}+\mathbf{d}_{\alpha}\left(\omega_{1}\right)-\mathbf{d}_{\beta}\left(\omega_{2}\right)$ is the vector that connects site $\alpha$ on molecule 1 with site $\beta$ on molecule 2, $r_{12}=\left|\mathbf{r}_{12}\right|$ is the distance between the centers of molecules 1 and 2, $\omega_{i}$ is the orientation of the molecules $i, \mathbf{d}_{\alpha}$ is the vector from the molecular center to site $\alpha$, see also figure 1 of [49]. Each of the off-center attraction sites is located at a distance $d_{\mathrm{s}}$ from the particles' center, $d_{\mathrm{s}}=\left|\mathbf{d}_{\alpha}\right|(\alpha=\mathrm{A}, \mathrm{B}, \mathrm{C}, \mathrm{D})$. In the model in question, only the site-site association $\mathrm{AC}, \mathrm{BC}, \mathrm{AD}$, and $\mathrm{BD}$ is allowed, all association 
energies are equal. Specifically, the interaction between sites is given as

$$
u_{\alpha \beta}\left(\mathbf{r}_{\alpha \beta}\right)= \begin{cases}-\varepsilon_{\mathrm{as}}, & \text { if } 0<\left|\mathbf{r}_{\alpha \beta}\right| \leqslant r_{\mathrm{c}} \\ 0, & \text { if }\left|\mathbf{r}_{\alpha \beta}\right|>r_{\mathrm{c}}\end{cases}
$$

where $\varepsilon_{\mathrm{as}}$ is the depth of the association energy well and $r_{\mathrm{c}}$ is the cut-off range of the associative interaction. Note that the same model was also used in several works.

The non-associative part of the pair potential, $u_{\mathrm{ff}}(r)$, is given as,

$$
u_{\mathrm{ff}}(r)=u_{\mathrm{hs}, \mathrm{ff}}(r)+u_{\mathrm{att}, \mathrm{ff}}(r),
$$

where $u_{\mathrm{hs}, \mathrm{ff}}(r)$ and $u_{\mathrm{att}, \mathrm{ff}}(r)$ are the hard-sphere (hs) and attractive (att) pair interaction potential, respectively. The hs term is,

$$
u_{\mathrm{hs}, \mathrm{ff}}(r)= \begin{cases}\infty, & \text { if } r<\sigma, \\ 0, & \text { if } r \geqslant \sigma,\end{cases}
$$

where $\sigma$ is the hs diameter. The attractive interaction is described by the square-well ( $\mathrm{SW}$ ) potential,

$$
u_{\text {att, ff }}(r)= \begin{cases}0, & \text { if } r<\sigma, \\ -\varepsilon, & \text { if } \sigma \leqslant r<\lambda_{\mathrm{ff}} \sigma, \\ 0, & \text { if } r \geqslant \lambda_{\mathrm{ff}} \sigma,\end{cases}
$$

where $\varepsilon$ and $\lambda_{\mathrm{ff}}$ are the depth and the range of the potential, respectively.

The pore walls are located at $z=-H / 2$ and $z=H / 2$. The external potential, $v(z)$, exerted on a fluid particle inside the pore by the "bare" (non-modified) walls is,

$$
v_{\mathrm{f}}(z)=v_{\mathrm{fw}}(H / 2+z)+v_{\mathrm{fw}}(H / 2-z) \text { for }-H / 2 \leqslant z \leqslant H / 2 .
$$

The function $v_{\mathrm{fw}}(z)$ is given by the Steele's 10-4-3 gas-solid potential [51, 52],

$$
v_{\mathrm{fw}}(z)=\varepsilon_{\mathrm{fw}}\left[\frac{2}{5}\left(\frac{\sigma_{\mathrm{fw}}}{z}\right)^{10}-\left(\frac{\sigma_{\mathrm{fw}}}{z}\right)^{4}-\frac{\sigma_{\mathrm{fw}}^{4}}{3 \Delta(z+0.61 \Delta)^{3}}\right],
$$

where $\varepsilon_{\mathrm{fw}}, \sigma_{\mathrm{fw}}$ are the energy and the size parameters describing fluid-wall interaction ( $\Delta$ is the solid inter-layer spacing).

In order to describe the layer of tethered chain molecules at each wall we use the approach presented already in [53-55]. The grafted layer at one wall is composed of chains of $M_{1}$ tangentially jointed segments, while each chain attached to the second wall comprises $M_{2}$ segments. For simplicity, we assume that the diameter of all the segments is the same and equal to $\sigma_{\mathrm{cc}}$. Moreover, we restrict ourselves to a symmetric case $M_{1}=M_{2}=M$.

The connectivity of segments in the chain $I$ is provided by imposing the bonding potential, $V_{\mathrm{b}}^{(I)}$, see [36, 37]

$$
\exp \left[-V_{\mathrm{b}}^{(I)}(\mathbf{R}) / k T\right]=\prod_{i=1}^{M_{I}-1} \delta\left(\left|\mathbf{r}_{i+1}-\mathbf{r}_{i}\right|-\sigma_{\mathrm{cc}}\right) / 4 \pi\left(\sigma_{\mathrm{cc}}\right)^{2},
$$

where $I=1,2 ; \mathbf{R}_{k} \equiv\left(\mathbf{r}_{1}, \mathbf{r}_{2}, \ldots, \mathbf{r}_{M}\right)$ is the vector that specifies the coordinates of all segments and $\delta(x)$ the Dirac function.

The first segment of the chain $I=1$ is bonded to the wall at $z=-H / 2$, whereas the first segment of the chain $I=2$ is bonded to the wall at $z=H / 2$. It means that these segments are fixed in the $z$ plane at the positions $z=\sigma_{\mathrm{cc}} / 2-H / 2$ and $z=H / 2-\sigma_{\mathrm{cc}} / 2$, respectively, or that the corresponding Boltzmann factors associated with the external potential are as follows

$$
\exp \left[-v_{\mathrm{s} 1}^{(1)}(z) / k T\right]=\delta\left(z-\left(\sigma_{\mathrm{cc}} / 2-H / 2\right)\right),
$$

and

$$
\exp \left[-v_{\mathrm{s} 1}^{(2)}(z) / k T\right]=\delta\left(z-\left(H / 2-\sigma_{\mathrm{cc}} / 2\right)\right)
$$


All the remaining segments of grafted chains are assumed to interact with the pore walls similarly to fluid species, namely,

$$
v_{\mathrm{s} i}^{(I)}(z)= \begin{cases}\infty, & z<-H / 2+\sigma_{\mathrm{cc}} / 2, \quad z>H / 2-\sigma_{\mathrm{cc}} / 2, \\ v_{\mathrm{s}}(z), & -H / 2+\sigma_{\mathrm{cc}} / 2 \leqslant z \leqslant H / 2-\sigma_{\mathrm{cc}} / 2,\end{cases}
$$

where the function $v_{\mathrm{s}}(z)$ is given by equation $(6)$, though with the parameters $\varepsilon_{\mathrm{bw}}, \sigma_{\mathrm{bw}}$, to abbreviate brush-wall interaction. In the present contribution, we assume $v_{\mathrm{s}}(z)=0$. This setup for segment-phobic walls makes the model simpler and makes interpretation of the results more transparent. Possible effects of attraction between segments and pore walls on the properties of the systems in question will be considered elsewhere.

The inter-particle interaction between all segments as well as between adsorbate molecules and each segment of the grafted chain is assumed in the form of the SW potential like it is given by equations (3)(5) with size and energy parameters equal to $\sigma_{\mathrm{cc}}, \lambda_{\mathrm{bb}}$ and $\varepsilon_{\mathrm{bb}}, \sigma_{\mathrm{bf}}, \lambda_{\mathrm{bf}}$ and $\varepsilon_{\mathrm{bf}}$, respectively. The presence of attraction between segments makes the grafted polymer layers thermal on their own.

\section{Theory}

The system is studied using the version of the density functional theory (DF), described already in detail in [36, 37, 53-55]. To avoid unnecessary repetition, we recall only the basic equations. A few additional equations are given in the supplementary material section.

Let us introduce the following notation. The symbols $\rho^{(\mathrm{cI})}(\mathbf{R})$ and $\rho(\mathbf{r})$ denote the local density of the chains tethered at the wall $(I=1,2)$ and of the fluid, respectively. We also define the local densities of consecutive chains' segments, $\rho_{\mathrm{s} j}^{(I)}(\mathbf{r})$, and the total segment density, $\rho_{\mathrm{s}}(\mathbf{r})$, as in [36],

$$
\rho_{\mathrm{s}}^{(I)}(\mathbf{r})=\sum_{j=1}^{M} \rho_{\mathrm{s} j}^{(I)}(\mathbf{r})=\sum_{j=1}^{M_{I}} \int \mathrm{d} \mathbf{R} \delta\left(\mathbf{r}-\mathbf{r}_{j}\right) \rho^{(\mathrm{cI})}(\mathbf{R}),
$$

where $\mathbf{R} \equiv\left(\mathbf{r}_{1}, \mathbf{r}_{2}, \ldots, \mathbf{r}_{M}\right)$ is a set of coordinates describing the positions of all segments of a given chain molecule. In the model with the external potential dependent solely on the distance from the pore walls and under the assumption of random distribution of tethered segments, the density profiles are one-dimensional.

Before proceeding to the essence of the theory, it is worth to make the following comments. In the experimental setup, the polymer brushes are prepared through two techniques referred to as as "grafting-to" and "grafting-from". The grafting-to method involves adsorption of pre-formed polymers to a substrate. On the other hand, in the grafting-from method, the polymer chains grow from the substrate modified by a certain initiator [6, 7]. In our gedankenexperiment mimicking grafting-from setup, the grafted polymer layers are present in the pore prior to water adsorption. Thus, a combined system, slit-like pore with tethered chains and with adsorbed fluid, is in equilibrium with the external reservoir containing solely a fluid at a given chemical potential or equivalently at a certain external pressure.

Consequently, in order to evaluate the density profiles we minimize the functional of the following thermodynamic potential [54, 55],

$$
\mathcal{Y}=F\left[\rho^{(\mathrm{c} 1)}(\mathbf{R}), \rho^{(\mathrm{c} 2)}(\mathbf{R}), \rho(\mathbf{r})\right]+A_{\mathrm{s}} \int \mathrm{d} z \rho(z)\left[v_{\mathrm{f}}(z)-\mu\right],
$$

where $F\left[\rho^{(\mathrm{c} 1)}(\mathbf{R}), \rho^{(\mathrm{c} 2)}(\mathbf{R}), \rho(\mathbf{r})\right]$ is the Helmholtz free energy functional and $\mu$ is the chemical potential of the fluid. The external field, $v_{\mathrm{f}}(z)$, is given by equation $[6$ and $\mu$ is the chemical potential. Minimization of the thermodynamic potential $\mathcal{Y}$ is performed under the constraint that the amount of tethered chains at each wall is constant,

$$
\int \mathrm{d} \mathbf{r} \rho_{\mathrm{s} 1}^{(I)}(z)=A_{\mathrm{s}} R_{\mathrm{c}}^{(I)}
$$


where $A_{\mathrm{S}}$ is the surface area and $R_{\mathrm{c}}^{(I)}$ is the brush density at the wall $(I=1,2)$. For simplicity, we restrict ourselves to symmetric systems with $R_{\mathrm{c}}=R_{\mathrm{c}}^{(1)}=R_{\mathrm{c}}^{(2)}$.

The free-energy functional consists of the ideal, $F_{\text {id }}$, and the excess, $F_{\text {ex }}$, term. The excess free energy is expressed as the sum of contributions arising from different kinds of interactions in the system [56[58]. The definition of the free energy of the system is given in the supplementary material. We only mention that the excess contribution due to chemical association is managed by using Wertheim's theory of association [59-62]. It involves the function $\chi_{\mathrm{A}}(z)$, the density profile of the fraction of molecules at the $z$ position that are not bonded at the site A, which is a local analogue of the mass action law for inhomogeneous chemically associating fluids.

The density profiles are obtained from minimization of the functional $\mathcal{Y}$. Minimization is carried out under the constraint given by equation (14).

$$
\frac{\delta \boldsymbol{Y}}{\delta \rho^{(\mathrm{c} 1)}(\mathbf{R})}=\frac{\delta \boldsymbol{y}}{\delta \rho^{(\mathrm{c} 2)}(\mathbf{R})}=0, \quad \frac{\delta \boldsymbol{y}}{\delta \rho(z)}=0 .
$$

Final equations for the density profiles are given in the supplementary material.

The vapour-liquid coexistence for a fluid confined in slit-like pores is found from the condition of equality of the thermodynamic potential $\mathcal{Y}$ for two different density profiles at a fixed temperature and chemical potential (cf. [54, 55]). The average densities of the coexisting phases of the fluid confined in a pore, $\langle\rho\rangle$, and the average fraction of non-bonded fluid particles, $\left\langle\chi_{\mathrm{A}}\right\rangle$, are calculated from the density profiles as

$$
\langle\rho\rangle=\frac{1}{H} \int_{-H / 2}^{H / 2} \rho(z) \mathrm{d} z, \quad\left\langle\chi_{\mathrm{A}}\right\rangle=\frac{1}{H} \int_{-H / 2}^{H / 2} \chi_{\mathrm{A}}(z) \mathrm{d} z,
$$

where $H$ is the pore width. Moreover, we explore the brush height, $\left\langle h_{\mathrm{b}}^{*}\right\rangle$, defined as in [17, 63],

$$
\left\langle h_{\mathrm{b}}^{*}\right\rangle=2 \frac{\int \mathrm{d} z z \rho_{\mathrm{s}}(z)}{\int \mathrm{d} z \rho_{\mathrm{s}}(z)} .
$$

\section{Results and discussion}

\subsection{Parameters of the model}

In this work, without loss of generality, we employ solely one water-like model designated as W1 in [43]. The model is characterized by the diameter of a particle, $\sigma=3.0342 \AA$, the depth and range of the attractive potential, $\varepsilon / k=250 \mathrm{~K}$ and $\lambda_{\mathrm{ff}}$, the association energy, $\varepsilon_{\mathrm{as}} / k=1400 \mathrm{~K}$, and the cut-off distance of the attractive site-site potential, $r_{\mathrm{c}}=2.10822 \AA$.

In order to avoid cumbersome notations, let us introduce the species subscripts $i, j$, referring to the brushes grafted at two walls, $i, j=1,2$, and to a fluid, if $i, j=3$. Thus, according to the notation introduced in the description of the model, we deal with: $\sigma_{33}=\sigma, \varepsilon_{33}=\varepsilon, \lambda_{33}=\lambda_{\mathrm{ff}}=1.7889$ (for W1 model). As concerns the brush and cross brush-fluid interactions, we choose $\sigma_{\mathrm{cc}}=\sigma, \lambda_{i j}=\lambda_{\mathrm{bb}}=1.4, \varepsilon_{i j}=\varepsilon_{\mathrm{bb}}=\varepsilon$ for $i, j=11,12,21$ and 22. Next, $\sigma_{i 3}=\sigma_{3 i}=\sigma, \varepsilon_{i 3}=\varepsilon_{3 i}=\varepsilon_{\mathrm{bf}}=\varepsilon$ and $\lambda_{i 3}=\lambda_{3 i}=\lambda_{\mathrm{bf}}=1.4$ for $i=1$ or 2 .

The fluid parameters, $\sigma$ and $\varepsilon$ are chosen as the length and energy units, respectively. The dimensionless quantities, such as diameters, pore width and energy of interactions, are marked by an asterisk. The dimensionless temperature, $T^{*}$, is defined as common, $T^{*}=k T / \varepsilon$.

In all figures, we used the reduced temperature, $T_{\mathrm{r}}^{*}, T_{\mathrm{r}}^{*}=T^{*} / T_{\mathrm{cb}}^{*}$, where $T_{\mathrm{cb}}^{*}$ is the critical temperature of the bulk water-like $\mathrm{W} 1$ fluid model $\left(T_{\mathrm{cb}}^{*}=2.72\right)$. The energy of interaction of water-like species with the pore walls is denoted as $\varepsilon_{\mathrm{fw}}^{*}$. As it is mentioned above, the segments of chains are just confined in the pore, but do not interact with the walls, i.e. $\varepsilon_{\mathrm{bw}}^{*}=0$, throughout this study. 

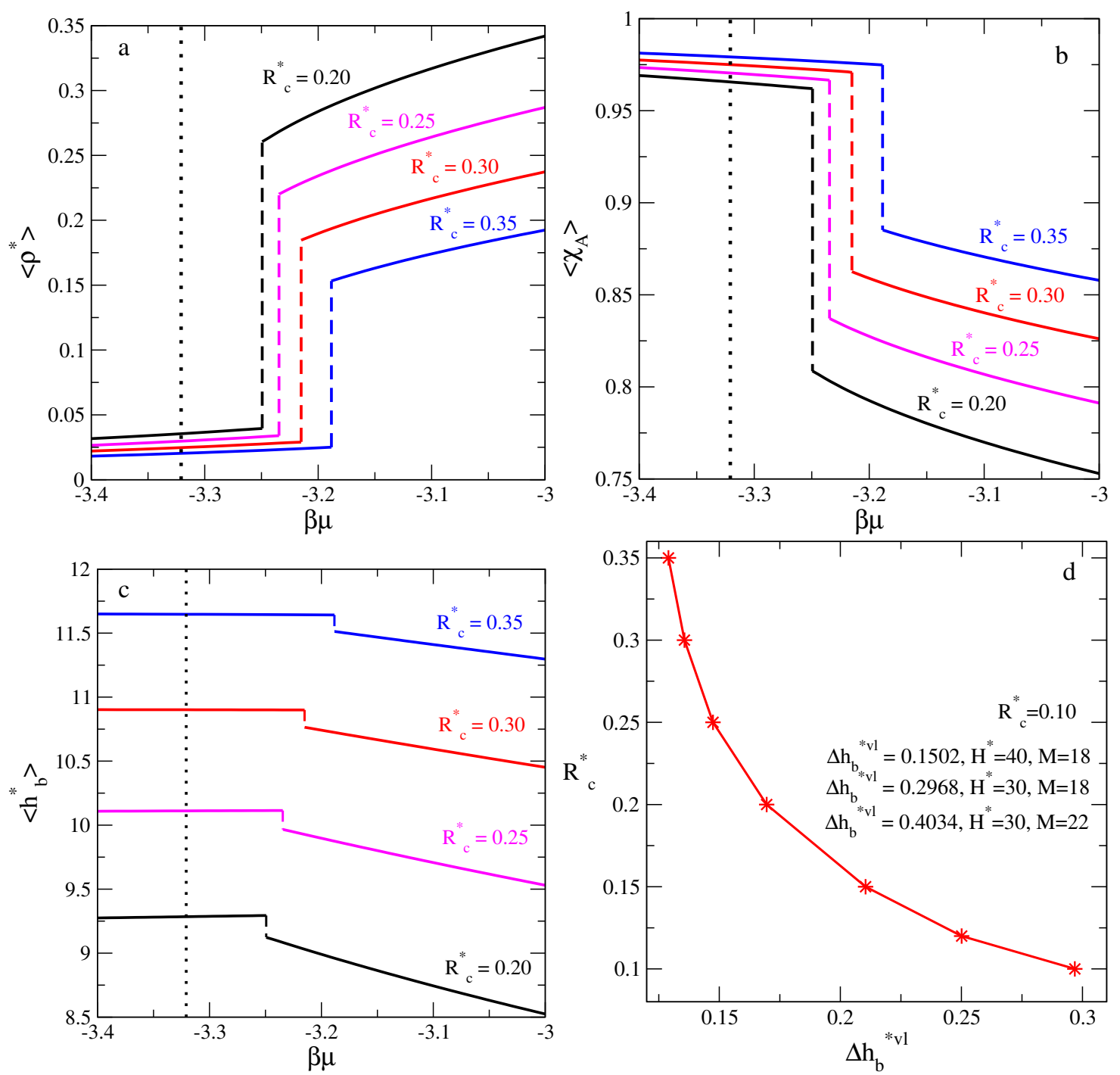

Figure 1. (Colour online) Adsorption isotherms of the $\mathrm{W} 1$ water model in the slit-like pore of the width, $H^{*}=30$, at temperature, $T_{\mathrm{r}}^{*}=0.85$, upon changing the grafted polymer layer density, $R_{\mathrm{c}}^{*}$. Panels (a), (b) and (c) refer to the projections, $\beta \mu-\left\langle\rho^{*}\right\rangle, \beta \mu-\left\langle\chi_{\mathrm{A}}\right\rangle$ and $\beta \mu-\left\langle h_{\mathrm{b}}^{*}\right\rangle$, respectively $(M=18)$. Dotted line in panels (a), (b) and (c) corresponds to the chemical potential at VL coexistence for the bulk fluid $\left(\beta \mu_{0}=-3.3208\right.$ at $\left.T_{\mathrm{r}}^{*}=0.85\right)$. Panel (d): Change of the brush height upon VL transition at different $R_{\mathrm{c}}^{*}$. In all panels: $\varepsilon_{\mathrm{fw}}^{*}=8.311, \varepsilon_{\mathrm{bb}}^{*}=\varepsilon_{\mathrm{bf}}^{*}=1, \lambda_{\mathrm{bb}}=\lambda_{\mathrm{fb}}=1.4$.

\subsection{Results}

The solution of equations for the density profiles permits to construct the adsorption isotherms and further discuss the phase diagrams. A set of results reported in figure 1 was obtained at different brush density, $R_{\mathrm{c}}^{*}$, for chains with $M=18$ in a quite wide pore $H^{*}=30$. The attraction strength between fluid species and pore walls is at $\varepsilon_{\mathrm{fw}}^{*}=8.311$, similar to the previous studies [42]. The pore walls are rather strongly attractive with this $\varepsilon_{\mathrm{fw}}^{*}$. The adsorption isotherms of the W1 water model in terms of average fluid density in the pore on chemical potential at a fixed $T_{\mathrm{r}}^{*}=0.85$ are shown in panel (a) of figure 1 .

The isotherms describe the vapour-liquid phase transition of a fluid in the pore and all of them exhibit hysteresis. However, only stable branches are plotted in the figure. The magnitude of the average density jump decreases as the brush density, $R_{\mathrm{c}}^{*}$, increases. As was discussed in figure 1 (a) of [42], capillary condensation, $\mu<\mu_{0}$, in the pore is observed in the absence of grafted chains on the walls whereas the 
capillary evaporation, $\mu>\mu_{0}$, is induced by the presence of grafted chains ( $\mu_{0}$ refers to the chemical potential value at bulk coexistence). The vapour density remains almost constant with the augmenting chemical potential up to the equilibrium phase transition point in the pore. On the other hand, trends of the behaviour of liquid density after transition are determined by the brush density. If the brush density is low, i.e., there is enough space in the pore for fluid adsorption, then the liquid density visibly grows upon increasing the chemical potential. However, at a high brush density, e.g., $R_{\mathrm{c}}^{*}=0.35$, the liquid density branch is less affected by the changes of chemical potential. In other words, the inclination of the liquid branch is determined by the $R_{\mathrm{c}}^{*}$ value.

A set of projections of adsorption isotherms in terms of $\left\langle\chi_{\mathrm{A}}\right\rangle-\beta \mu$ is shown in figure 11 (b). Here, the $\left\langle\chi_{\mathrm{A}}\right\rangle$ values corresponding to vapour remain almost constant and close to unity upon increasing the chemical potential up to the phase transition point. Thus, the vapour phase at each $R_{\mathrm{c}}^{*}$ is predominantly composed of non-bonded molecules. The fraction of non-bonded particles in liquid phases is much lower. The magnitude of the jump of $\left\langle\chi_{\mathrm{A}}\right\rangle$ at the transition decreases upon increasing $R_{\mathrm{c}}^{*}$, actually determined by the jump of the adsorbed fluid density.

Changes of the height of the layer of tethered molecules upon the phase transition of the fluid in the pore are shown in terms of the average brush height, $\left\langle h_{\mathrm{b}}^{*}\right\rangle$ on $\beta \mu$ in figure 1 (c). The brush height jump is small upon the fluid phase transition. The $\left\langle h_{\mathrm{b}}^{*}\right\rangle$ is smaller in the liquid phase compared to the vapour phase at each $R_{\mathrm{c}}^{*}$. In the liquid phase, the average brush height decreases upon increasing the adsorbed fluid density. However, the inclination of the lines depends on $R_{\mathrm{c}}^{*}$. The polymer chains comprising the brush are much more extended at high values of $R_{\mathrm{c}}^{*}$, in comparison with low $R_{\mathrm{c}}^{*}$. Thus, the conditions of adsorption gedankenexperiment affect the brush stiffness. In order to appreciate the magnitude of the jump of $\left\langle h_{\mathrm{b}}^{*}\right\rangle$ upon changing $R_{\mathrm{c}}^{*}$, we have constructed a plot shown in panel (d) of figure 1

We observe that the change of the brush height upon VL transition $\left(\Delta h_{\mathrm{b}}^{* \mathrm{vl}}=\left\langle h_{\mathrm{b}}^{* \mathrm{~V}}\right\rangle-\left\langle h_{\mathrm{b}}^{* 1}\right\rangle\right)$ decreases as the grafting density, $R_{\mathrm{c}}^{*}$, increases. A smaller jump of $\Delta h_{\mathrm{b}}^{* \mathrm{vl}}$ at a high $R_{\mathrm{c}}^{*}$ is due to a smaller available volume for adsorption of fluid molecules and, consequently, to a weaker influence of fluid species on the elongation of the chains in a dense brush. Changes of the brush height upon VLE transition in the adsorbed fluid are most pronounced at quite low values of $R_{\mathrm{c}}^{*}$. One example concerns $R_{\mathrm{c}}^{*}=0.1$. We performed additional calculations for the same conditions $\left(R_{\mathrm{c}}^{*}=0.1, M=18\right)$, but in a wider pore $H^{*}=40$. Then, $\Delta h_{\mathrm{b}}^{* \mathrm{vl}}$ changes from 0.2968 for $H^{*}=30$ to 0.1502 for $H^{*}=40$. On the other hand, if we change the nominal change length from $M=18$ to $M=22$ at a fixed pore width, $H^{*}=30$ and at $R_{\mathrm{c}}^{*}=0.1$, then $\Delta h_{\mathrm{b}}^{* \mathrm{vl}}$ changes from 0.2968 to 0.4034 (see the inset in panel (d) of figure 1 ). Consequently, changes of brush elongation in terms of $\left\langle h_{\mathrm{b}}^{*}\right\rangle$ are sensitive to the pore width and to the length of the chains at a fixed temperature. This observation can have implications for the setup of systems with controllable thermodynamics of adsorption and fluid transport through nanochannels.

After evaluating various adsorption isotherms, we can construct the VL coexistence envelopes. In figure 2 (a), the effect of $R_{\mathrm{c}}^{*}$ on the $\left\langle\rho^{*}\right\rangle-T_{\mathrm{r}}^{*}$ projection of the coexistence at two values of the pore widths $H=20$, and $H=30$, is displayed. As concerns the effect of $R_{\mathrm{c}}^{*}$ at constant $H^{*}$, we note that the coexistence envelope shrinks with increasing $R_{\mathrm{c}}^{*}$. The liquid branch is much more affected by the value of $R_{\mathrm{c}}^{*}$ compared to the vapour branch density. The effect of $H^{*}$ at constant $R_{\mathrm{c}}^{*}$ can be summarized as follows. Upon increasing $H^{*}$, the phase diagram in the average density-temperature plane widens, principally due to the augmenting liquid density at coexistence. In general, shrinking of the coexistence envelope is observed upon the augmenting confinement due to a decreasing $H^{*}$ or increasing $R_{\mathrm{c}}^{*}$. In both cases, the critical temperature decreases very slightly. Still, it is close to the bulk critical temperature. The critical density decreases as well. Apparent difference of the critical density in the pores and in the bulk, as well as of the densities along coexistence, can be attributed in part to the normalization of $\left\langle\rho^{*}\right\rangle$ using $H^{*}$, in contrast to a common normalization of the adsorbed density through the available volume for adsorption in the theory of fluids in disordered porous media, see e.g., [64, 65].

In order to explore the effect of $R_{\mathrm{c}}^{*}$ and $H^{*}$ on the brush height, the $\left\langle h_{\mathrm{b}}^{*}\right\rangle-T_{\mathrm{r}}^{*}$ projections of the coexistence envelope are shown in figure 2 (b). At a fixed $R_{\mathrm{c}}^{*}$, either at 0.2 or 0.3 , if the pore width, $H^{*}$, increases from $H^{*}=20$ to $H^{*}=30$, the coexistence envelope changes from the thumb-like shape into ice-pick-like shape. A higher value of $\left\langle\rho^{*}\right\rangle$ along the liquid brunch of the coexistence in a pore $H^{*}=30$ compared to $H^{*}=20$ (cf. panel a of this figure), means that the fluid particles are confined to a smaller volume and, as a consequence, the $\left\langle h_{\mathrm{b}}^{*}\right\rangle$ value is higher in the pore $H^{*}=30$ compared to $H^{*}=20$. In 


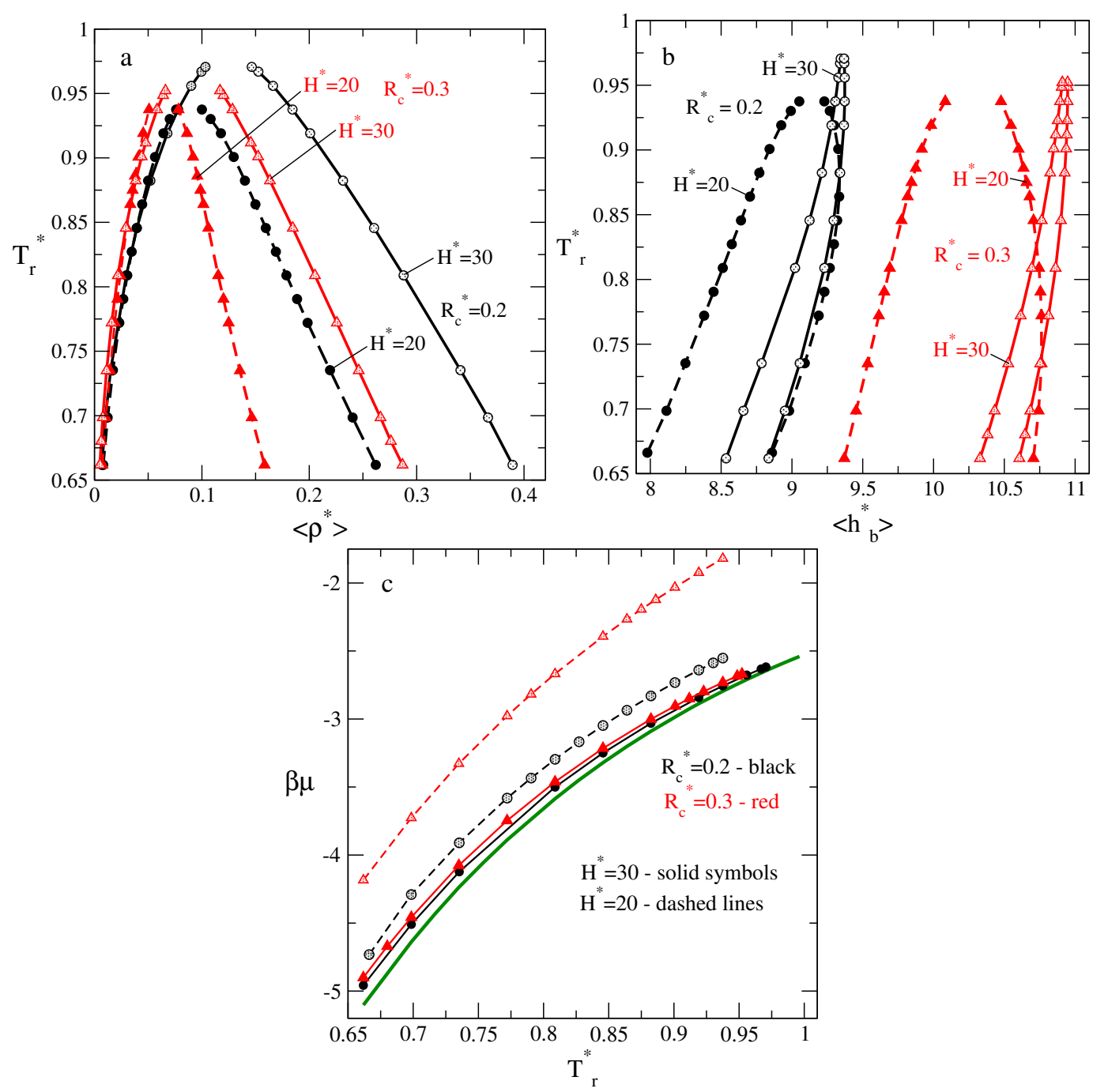

Figure 2. (Colour online) Projections of the VL coexistence envelope for $\mathrm{W} 1$ water model upon changing the tethered polymer layer density, $R_{\mathrm{c}}^{*}$ and pore width, $H^{*}$. Panels (a), (b) and (c) refer to $\left\langle\rho^{*}\right\rangle-T_{\mathrm{r}}^{*}$, $\left\langle h_{\mathrm{b}}^{*}\right\rangle-T_{\mathrm{r}}^{*}$ and $T_{\mathrm{r}}^{*}-\beta \mu$ projections, respectively. The nomenclature of lines and symbols is given in the figures. The bulk fluid coexistence is plotted as a thick green line in panel (c). All the parameters are as in figure 1 [panels (a), (b) and (c)].

other words, the brush, in contact with the liquid, is more "compressed" in a narrower pore compared to a wider pore. The effect of $R_{\mathrm{c}}^{*}$ at constant $H^{*}$ can be easily interpreted as well. A denser brush in terms of $R_{\mathrm{c}}^{*}$ leads to a higher $\left\langle h_{\mathrm{b}}^{*}\right\rangle$ due to augmenting effects of excluded volume for the inner parts of chains corresponding to the brush body.

Finally, we plotted the $T_{\mathrm{r}}^{*}-\beta \mu$ projection of the coexistence envelopes. The curves shown in figure 2 (c) describe the capillary evaporation in the entire temperature interval. The chemical potential at transition in the pore becomes closer to the bulk coexistence if the pore width increases. Moreover, in the wide pore, $H^{*}=30$, the transformation of vapour into liquid occurs at a very similar value of the chemical potential for two values of $R_{\mathrm{c}}^{*}$, namely at 0.2 and 0.3 . In a narrower pore, $H^{*}=20$, though the difference of the values of chemical potential at transition is substantial.

An overall thermodynamic picture described in the previous figures follows from the distribution of species in the pore and in the resulting interface between brush and fluid. Therefore, it is of importance to discuss the density profiles under different conditions. The density profiles of grafted polymer layers and of a fluid, related to the present study, were reported previously in several works. It is well known 
from the self-consistent field theory that the system of grafted chains at moderate and high coverage is characterized by the density profile of parabolic shape [25], in agreement with findings by other methods and experimental observations. On the other hand, the density profiles for grafted species were obtained using computer simulations (in the framework of molecular dynamics and dissipative particle dynamics techniques) [15-[18]. Specifically, the profiles reported from the laboratory of Binder refer to the model with implicit solvent or to the model of a grafted layer in the presence of a hard sphere fluid only. Moreover, the results concern either a single flat substrate or cylindrical pores of various diameters. The slit-like pore setup was considered in [15]. We are not aware of simulations data for systems that involve fluids with attractive interactions, either non-associative or with chemical association. Therefore, the density profiles obtained by using DF methodology are discussed with respect to computer simulations results at the level of trends observed within both methods.

Examples of the density profiles for fluid species and for the brush at vapour-liquid coexistence for fluid species are presented in figure 3. The fluid density profile in the panels (a) and (b) of this plot corresponds to a liquid state. Only one part of the profiles is shown due to the symmetry of distributions w.r.t. pore center in panel (a). From figure 3 (a), we learn that the fluid density in the center of a wide pore
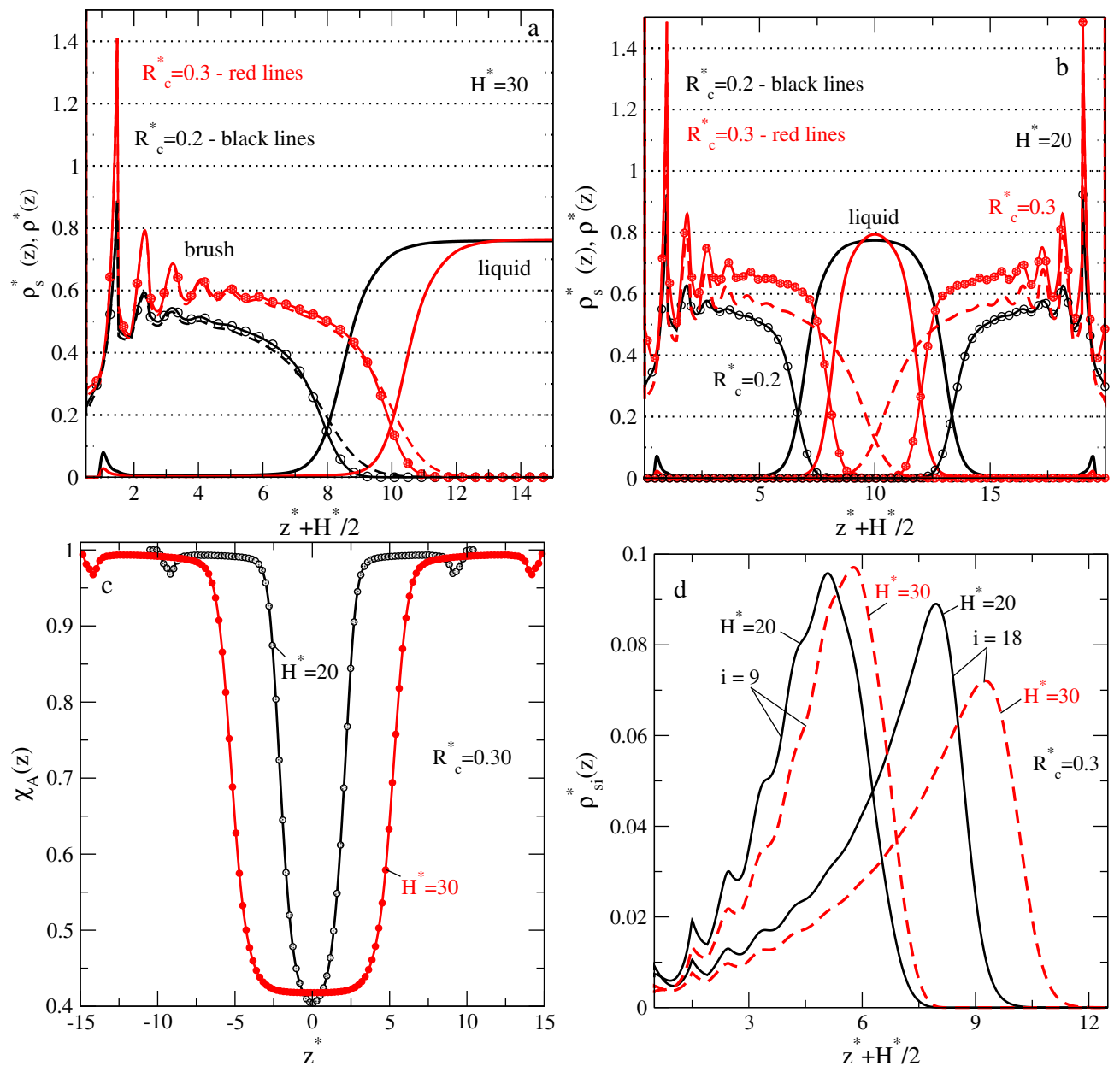

Figure 3. (Colour online) Examples of the fluid density profiles and brush density profiles [panels (a) and (b)], and the density profiles for the non-bonded fluid species [panel (c)] of thermal tethered polymer layer and of W1 fluid model at VL coexistence for $R_{\mathrm{c}}^{*}=0.2$ and 0.3 . Panel (d): The density profiles of selected segments $i$ (marked in the figure) of grafted chains at fluid VL coexistence. The dashed lines in panels (a) and (b) represent the brush profiles in contact with fluid vapour at VL coexistence. The pore width and the number of segments in grafted chain molecules are $H^{*}=20$ and $30, M=18, T_{\mathrm{r}}^{*}=0.735$. All other parameters are as in figure 1 [panels (a)-(c)]. 
$\left(H^{*}=30\right)$ is practically constant and takes the value very close to the density of the bulk W1 water model at coexistence for the temperature in question $\left(\rho_{\mathrm{b}}^{*}=0.7716\right)$. Upon increasing the surface coverage of grafted chains, $R_{\mathrm{c}}^{*}$, from 0.2 to 0.3 , the brush layer becomes wider, indicating the stretching of chains. This behaviour is in accordance with what is observed from the analysis of computer simulation results, see for example panels (c) and (e) of figure 5 from [18]. A similar trend of behaviour with increasing the surface coverage is illustrated in figure 4 of [15]. Augmenting $R_{\mathrm{c}}^{*}$ leads to a well packed structure of the inner part of the grafted chains layer. A part of the density profile, $\rho_{\mathrm{s}}^{*}(z)$, describing the external part of the polymer layer exposed to the adsorbed liquid decays similarly for two values of the surface coverage considered in figure 3 (a). Apparently, the stretching of chains is a quite strong effect, because the fluid occupies less space in the central part of the pore at $R_{\mathrm{c}}^{*}=0.3$ compared to $R_{\mathrm{c}}^{*}=0.2$ [figure 3 (a)]. Consequently, the average fluid density in the pore is lower in the former case compared to the latter, in accordance with the phase coexistence envelopes in figure 2(a). Moreover, the profiles of brushes in figure 3 (a) exhibit a collapse effect upon the fluid transition from vapour to liquid, cf. dashed (brush in contact with vapour) and solid lines in figure 3 (a), in accordance with the corresponding phase diagrams. This effect is intrinsically out of reach for the model considered in simulations in [16].

In general terms, the shape of the interface between brush and liquid in a pore with $R_{\mathrm{c}}^{*}=0.2$ and $R_{\mathrm{c}}^{*}=0.3$, as well as its width are similar. The fluid species in fact permeate only a small outer part of each brush. In contrast to this outer part, where the brush density profile decays, the inner part exhibits oscillations that develop with increasing $R_{\mathrm{c}}^{*}$. These oscillations witness a dense packing of brush segments. A small probability to find fluid particles very close to the pore wall is nevertheless observed as a result of fluid-wall attraction.

Similar trends of behaviour of the profiles can be seen in figure 3 (b) for the case of a narrower pore, $H^{*}=20$. Apparently, the interface width is smaller. The inner parts of the brush profiles become flatter compared to panel (a), indicating a higher compression of the brushes in this pore $\left(H^{*}=20\right)$ in comparison with $H^{*}=30$. This behaviour has its origin in the strong collapse of the brush layers at two walls upon the fluid transition from vapour to liquid. The brushes in contact with vapour [dashed lines in figure 3(b)] exhibit a high degree of interdigitation, see [42] for a detailed description of this phenomenon within DF approach. By contrast, the liquid phase is dense and vigorously separates brushes from two walls. The magnitude of the observed changes depends on the pore width and grafted layer density. As concerns this kind of trends described by computer simulations, we refer to figure 4 of [15], where the interdigitation of brushes is the result of augmenting density of grafted chains.

The effect of $H^{*}$ on the density profile of fluid species non-bonded at a site, $\chi_{\mathrm{A}}(z)$, is shown in figure $3(\mathrm{c})$. The fraction of non-bonded particles, $\chi_{\mathrm{A}}(z)$, is low in the central part of the pore due to a high fluid density there. The density profile, $\chi_{\mathrm{A}}(z)$, shrinks as the pore becomes narrower. The width of interface in terms of $\chi_{\mathrm{A}}(z)$ follows the trends of the fluid density in this part of the pore.

An additional insight into the mechanism of "compression" of the brush upon changing the pore width is given in figure $3(\mathrm{~d})$. We show the changes of the density profiles of selected segments, $\rho_{\mathrm{s} i}^{*}(z)$, of chain molecules comprising the brush. The inner segments, $i<9$, behave similarly in both cases, $H^{*}=20$ and $H^{*}=30$. The density profile of a middle segment, $i=9$, exhibits a shift to the wall for $H^{*}=20$ compared to $H^{*}=30$, still the height of the maximum is almost equal in both cases. More pronounced changes occur for the density profiles of segments exposed to the liquid phase. In particular, the density profile of the terminating segment, $i=18$, is much closer to the pore wall in the case $H^{*}=20$ in comparison with $H^{*}=30$. Moreover, the maximum of this profile is much higher for $H^{*}=20$, if we compare with $H^{*}=30$.

Our discussion of the density profiles of species with respect to the conclusions reached by computer simulations of similar class of models would be incomplete without describing the changes of the profiles on the number of segments of grafted chains. Some insights into this issue are provided in figure 4. Namely, one can observe that the height of the brush layer increases with increasing the chain length, provided the grafting density is kept constant, figure 4(a). This behaviour from the DF theory is in agreement with the trends coming from computer simulations with implicit solvent, cf. figure 5 of [18]. Again, shrinking of the grafted polymer layers can be observed for models with different $M$ upon fluid phase transition from vapour to liquid, figure 4 (a). Stretching of chains, at constant $R_{\mathrm{c}}^{*}$, is more pronounced with an increasing number of segments, cf. the density profiles of the last segments for the models with $M=14$ and $M=22$, figure 4 (b). A similar conclusion follows from simulations, see panels 

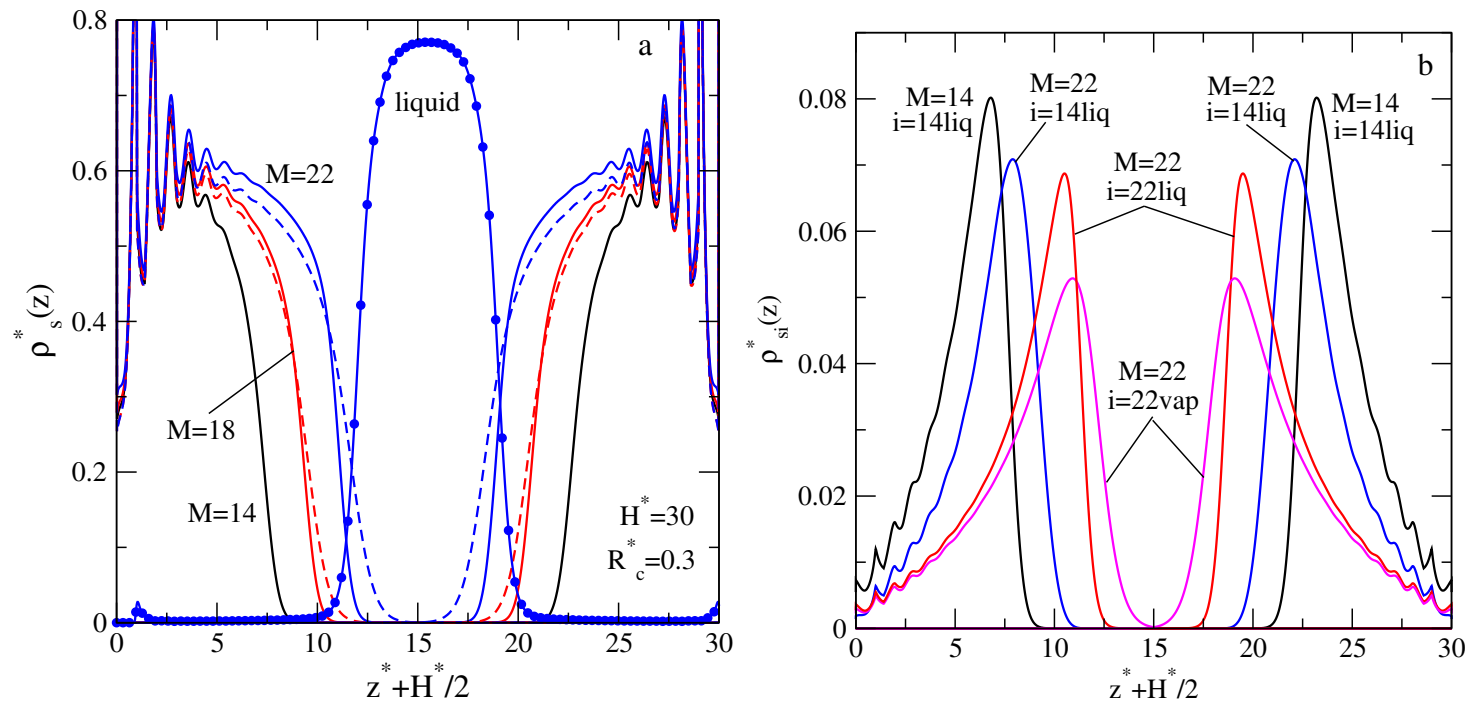

Figure 4. (Colour online) Effect of grafted chains length: examples of the brush density profiles and of the fluid distribution (for the model with $M=22$ ) in the pore [panel (a)], and the density profiles of selected segments $i$ (marked in the figure) of grafted chains at fluid VL coexistence. The dashed lines in panel (a) represent the brush profiles in contact with fluid vapour at VL coexistence. The segments in contact with fluid vapour or liquid in panel (b) are marked as ivap and iliq, respectively. The pore width is $H^{*}=30$ and the grafting density is at $R_{\mathrm{c}}^{*}=0.3, T_{\mathrm{r}}^{*}=0.735$. The number of segments in grafted chain is given in the figure. All other parameters are as in figure 1 [panels (a)-(c)].

(b), (d) and (f) of figure 5 from [18]. Moreover, essential changes of the profile describing the last, most exposed to the fluid body, segments, occur upon condensation in the pore, figure 4 (b).

All previous observations concerning the thermal response of brushes are concerned in one way or another with the vapour-liquid coexistence of the water-like fluid in the pores of different width and with different density of grafted polymer chains. In the last part of this communication, we would like to use a slightly different setup. Namely, we assume that the bulk fluid density is fixed at $\rho_{\mathrm{b}}^{*}=0.8$. Then, one
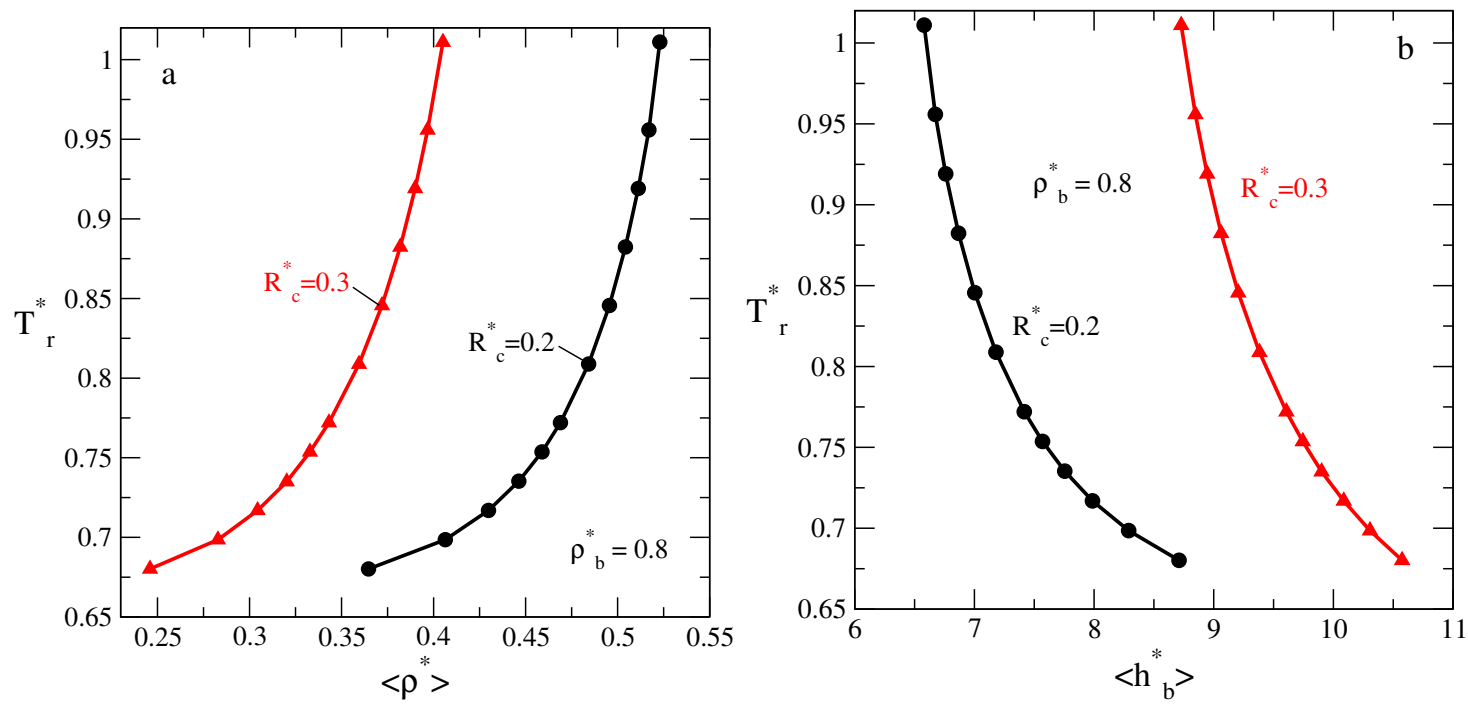

Figure 5. (Colour online) Density and brush height on temperature at a fixed bulk liquid density, $\rho_{\mathrm{b}}^{*}=0.8$, for $\mathrm{W} 1$ water model in a pore $H^{*}=30(M=18)$. Panels (a) and (b) refer to $\left\langle\rho^{*}\right\rangle-T_{\mathrm{r}}^{*}$ and $\left\langle h_{\mathrm{b}}^{*}\right\rangle-T_{\mathrm{r}}^{*}$ projections, respectively. All parameters are as in figure 1 
is able to follow the temperature trends of behaviour of the systems in question in an ample interval of temperatures starting for example from supercritical conditions down to $T_{\mathrm{r}}^{*} \approx 0.65$ remaining in the bulk liquid phase. We restrict ourselves to the pore with the width $H^{*}=30$ only in this part of the work.

Evolution of the average fluid density in the pore with temperature, at a fixed bulk fluid density, is shown in panel (a) of figure 5 for two systems with $R_{\mathrm{c}}^{*}=0.2$ and with $R_{\mathrm{c}}^{*}=0.3$. In both cases, $\left\langle\rho^{*}\right\rangle$ monotonously decreases with a decreasing temperature. This behaviour is most pronounced at low temperatures. Apparently, trends of behaviour of both curves in the figure are determined by the augmenting effects of attractive interactions while temperature goes down. The effect of $R_{\mathrm{c}}^{*}$ reduces only to the shift along the $\left\langle\rho^{*}\right\rangle$ axis. The average brush height, $\left\langle h_{\mathrm{b}}^{*}\right\rangle$, depends on temperature as well [figure 5(b)]. Namely, it increases with a decreasing temperature. This behaviour is straightforwardly correlated with the dependence of $\left\langle\rho^{*}\right\rangle\left(T_{\mathrm{r}}^{*}\right)$. When the averaged adsorbed density decreases, the average brush height increases. Two curves describing $\left\langle h_{\mathrm{b}}^{*}\right\rangle\left(T_{\mathrm{r}}^{*}\right)$ at different $R_{\mathrm{c}}^{*}$ behave similarly. Just in the case $R_{\mathrm{c}}^{*}=0.3$, a lower average density of adsorbed fluid (in comparison with $R_{\mathrm{c}}^{*}=0.2$ ) leads to higher values of the average brush height.
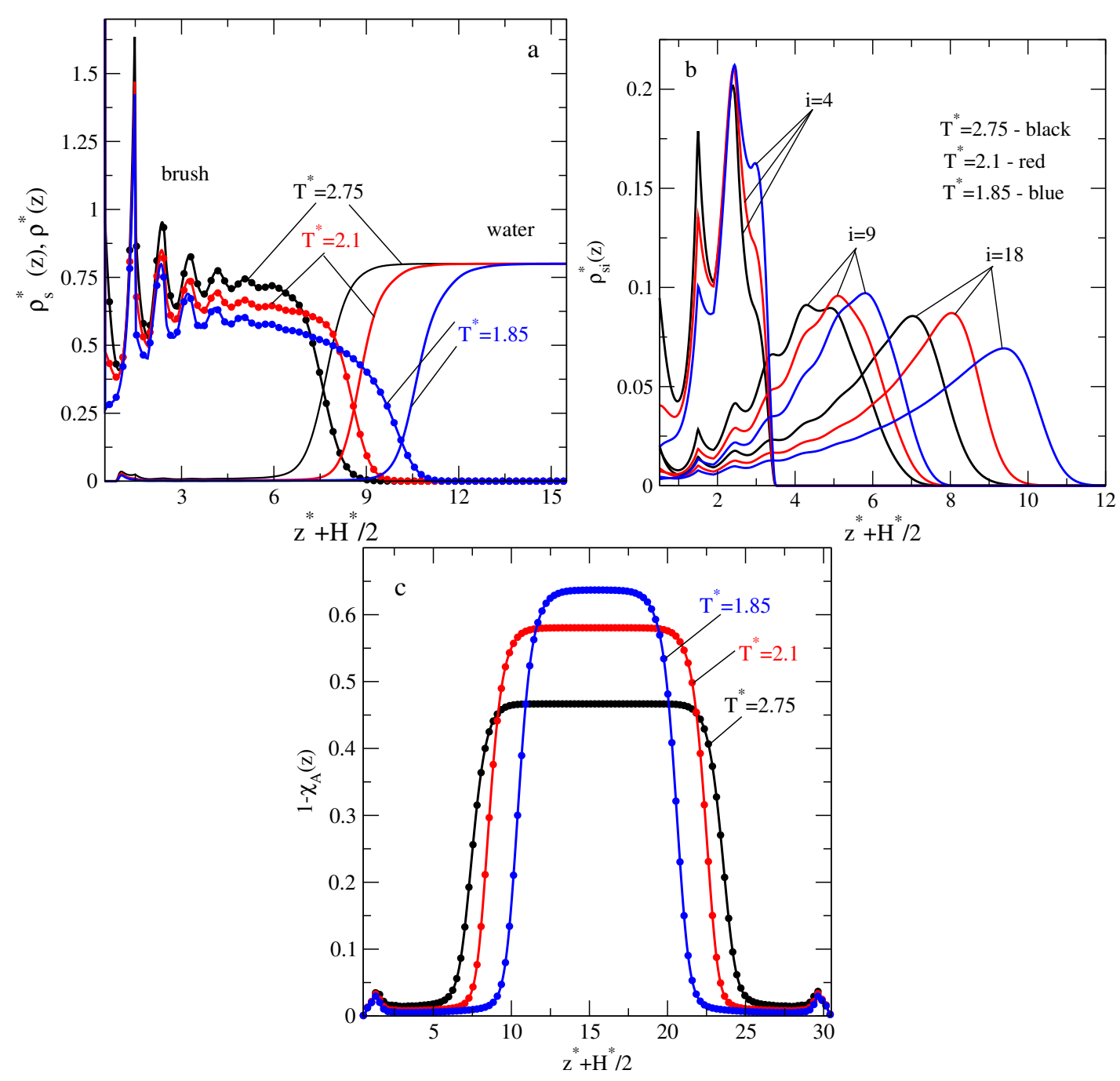

Figure 6. (Colour online) Examples of the liquid density profiles and brush density profiles [panel (a)], density profiles of selected segments $i$ [panel (b)], and density profiles of fluid species bonded at a site A [panel (c)] at different temperatures, $\rho_{\mathrm{b}}^{*}=0.8$. The pore width, the number of segments in grafted chain molecules and the tethered polymer layer density are $H^{*}=30, M=18$ and $R_{\mathrm{c}}^{*}=0.3$, respectively. All other parameters are as in figure 1 
It is of interest to find interpretation of this behaviour in terms of the microscopic structure of the fluid and brush in the pore and the interface between them. One set of examples is presented in figure 6 . From figure 6 (a) we learn that the brush density profile is essentially affected by temperature. It is stiffer at high temperature and much more diffused at a low temperature. However, the fluid permeates the brush body better at a high temperature. Apparently, the liquid slab at e.g., $T^{*}=2.75$ exerts a higher pressure over the brush due to a higher $\left\langle\rho^{*}\right\rangle$, cf. figure 5 (a), in comparison with e.g., $T^{*}=1.85$. Better insights into the changes of the brush height can be obtained from the analysis of the segment density profiles, figure 6(b), the inner part of the brush, i.e., for the segments number $i<9$ does not change much upon a decreasing temperature. This is well seen for example from the profile describing the segment $i=4$. The outer part of the brush described in terms of the distribution of segments with $i>9$ is much more affected by the temperature changes. The most pronounced effect is seen for the segment $i=18$ dangling quite freely in the fluid.

The structure of the liquid slab in the pore center can be analyzed in terms of the fluid density profiles shown in panel (a) and in terms of the degree of bonding between water-like particles. The liquid situated in the pore center is predominantly composed of fluid particles participating in bonds whereas water-like particles permeating the brush do not form bonds between them, figure 6 (c). How this distribution is manifested in the interface tension is an open question at the moment. However, it is worth mentioning that the width of the interface in terms of $\chi_{\mathrm{A}}(z)$ is sensitive to temperature as well.

\section{Summary and conclusions}

In this short report, we presented theoretical results from a version of the DF theory concerning adsorption of water into slit-like pores in which the walls are chemically modified by grafted chain molecules that form molecular brushes. The fluid model was taken from [43] and was incorporated into the DF approach. The model for the entire system involves the non-electrostatic interactions solely.

In contrast to our previous research [40-42], we included segment-segment attractive interaction in the form of a square well. This modification makes the brushes thermal on their own which leads to thermal responses due to fluid species and to the grafted chains. Apparently, the theory formulated in the spirit of semi-grand ensemble corresponds to the grafting from the procedure in the experiments because the bulk reservoir contains solely the fluid species.

The first setup employed here comprises the calculation of the thermodynamic potential at a given external chemical potential and external field, and its minimization to obtain the density profiles for adsorbed fluid and for grafted chains subsystem. We analysed and discussed a few examples of adsorption isotherms and of vapour-liquid coexistence envelopes for a confined fluid using average densitytemperature, chemical potential-temperature, chemical potential-brush height. On the other hand, we explored the microscopic structure of the interface between the brush and adsorbed fluid.

Capillary evaporation of water-like fluid model is observed at a high density of tethered chains, $R_{\mathrm{c}}^{*}$ in contrast to condensation in the absence of brushes in the pore. Upon fluid vapour-liquid transition, the brush height decreases via the jump. If the parameter $R_{\mathrm{c}}^{*}$ that describes the brush density is high, the grafted polymer chains attain an extended configuration leading to a high $\left\langle h_{\mathrm{b}}^{*}\right\rangle$ for the fluid in the vapour phase and a slightly smaller brush height. Under such a condition, the fluid weakly permeates the brush. A stronger brush collapse is expected at a lower tethered chains density.

The brush density profiles at high values of $R_{\mathrm{c}}^{*}$ have the shape that can be interpreted as a well structured, dense inner part and a more flexible outer part with dangling ends exposed to vapour or liquid. Upon vapour-liquid phase transition, this outer part suffers most pronounced changes. In addition, it is important to note that the predictions concerned with the behaviour of the density profiles from the DF methodology are in agreement with the trends observed by using computer simulations as tools [15. 18]. Complementary comparisons of the profiles from the DF approach for fluid-grafted layer of short chains with Monte Carlo simulations were also presented (though for different models of interactions) in [66].

On the other hand, we were interested in the exploration of temperature trends of behaviour of the system that are not related to the VL phase transition. With this purpose, we explored a setup at a constant bulk fluid density that permits to follow the thermal response of the system from the supercritical temperature down to $T_{\mathrm{r}}^{*} \approx 0.65$. 
A decreasing temperature leads to a lower averaged density of adsorbed species. Consequently, the average brush height monotonously increases. Swelling of the brush is due to the change of distribution of segments of grafted chains of the outer part of the brush.

Finally, possible extensions or improvements of the model and method should be attempted. As concerns the model, it would be of interest to investigate the effect of the block structure of grafted polymers that would interact differently with water-like particles. Furthermore, the adsorption of mixtures is worth to explore, evidently the condensation and mixing properties would change due to the presence of brushes [67]. In particular, if the demixing line is present in the phase diagram of the binary mixture, it would shift and possibly change its inclination due to the presence of brushes on the walls. In order to reach novel findings, however, one inevitably needs to employ a more sophisticated modelling of the system of grafted polymer molecules. Research along these lines is currently in progress in our laboratory and will be reported elsewhere.

\section{A. Helmholtz free energy functional}

The Helmholtz free energy is the sum of ideal and excess terms, $F=F_{\text {id }}+F_{\text {ex }}$, where the excess term is expressed as the sum of the contributions arising from different kinds of interactions in the system, $F_{\mathrm{ex}}=F_{\mathrm{hs}}+F_{\mathrm{c}}+F_{\mathrm{as}}+F_{\mathrm{att}}$, namely due to the volume exclusion, $F_{\mathrm{hs}}$, the connectivity of chains, $F_{\mathrm{c}}$, due to association of fluid molecules, $F_{\text {as }}$ and due to attractive van der Waals interactions, $F_{\text {att }}$. For the sake of brevity, we omitted the functional dependencies of all free energy terms.

The ideal part, $F_{\text {id }}$, is given by an exact expression [36]

$$
\begin{aligned}
\frac{F_{\mathrm{id}}}{k T}=\int \mathrm{d} \mathbf{R} \rho^{(\mathrm{c} 1)}(\mathbf{R}) V_{\mathrm{b}}^{(1)}(\mathbf{R}) / k T+\int \mathrm{d} \mathbf{R} \rho^{(\mathrm{c} 2)}(\mathbf{R}) V_{\mathrm{b}}^{(2)}(\mathbf{R}) / k T+\int \mathrm{d} \mathbf{R} \rho^{(\mathrm{c} 1)}(\mathbf{R})\left\{\ln \left[\rho^{(\mathrm{c} 1)}(\mathbf{R})\right]-1\right\} \\
+\int \mathrm{d} \mathbf{R} \rho^{(\mathrm{c} 2)}(\mathbf{R})\left\{\ln \left[\rho^{(\mathrm{c} 2)}(\mathbf{R})\right]-1\right\}+\int \mathrm{d} \mathbf{r} \rho(\mathbf{r})\left\{\ln \left[\rho(\mathbf{r}) \Lambda^{3}\right]-1\right\},
\end{aligned}
$$

where $\Lambda$ is thermal wavelength of fluid molecules.

The free energy due to hard-sphere interactions, $F_{\mathrm{hs}}$, is evaluated in the framework of the White Bear version of the fundamental measure theory [37, 57]. This approach requires introduction of four scalar, and two vector averaged densities. For the sake of brevity, the definitions of averaged densities are omitted, they are given by equations (3)-(6) of [37]. Since the fundamental measures theory is wellknown, we do not repeat it here. The definition for the hard-sphere contribution to the free energy is given by equations (1), (9), (10) and (11) of [37]. Similarly, the connectivity contribution to the Helmholtz free energy, $F_{\mathrm{c}}$, was reported in several works, see, e.g., equations (13) and (14) of [58].

The term arising from associative interactions between fluid molecules, $F_{\text {as }}$, results from the theory outlined in [38, 39, 56]

$$
F_{\text {ass }} / k T=4 \int \mathrm{d} \mathbf{r} n_{0}(z) \zeta(z)\left\{\ln \chi_{\mathrm{A}}(z)-\frac{1}{2}\left[\chi_{\mathrm{A}}(z)-1\right]\right\},
$$

where $\chi_{\mathrm{A}}(z)$ is the density profile of the fraction of molecules at position $z$ that are not bonded at the site $\mathrm{A}$,

$$
\chi_{\mathrm{A}}(z)=\left[1+n_{0}(z) \zeta(z) \sum_{\alpha \in \Gamma} \chi_{\alpha}(z) \Delta(z)\right]^{-1},
$$

and where $\Delta(z)$ describes the intermolecular site-site bonding, $\Delta(z)=4 \pi K F g_{\mathrm{hs}}\left(\sigma, n_{i}(z)\right)$, where $F=$ $\exp \left(\varepsilon_{\mathrm{as}} / k T\right)-1$. The parameters of the model determine the expression for site-site bonding volume, $K$ [49, 50],

$$
\begin{aligned}
K & =\frac{\sigma^{2}}{72 d_{\mathrm{s}}^{2}}\left\{\ln \left[\left(r_{\mathrm{c}}+2 d_{\mathrm{s}}\right) / \sigma\right]\left(6 r_{\mathrm{c}}^{3}+18 r_{\mathrm{c}}^{2} d_{\mathrm{s}}-24 d_{\mathrm{s}}^{3}\right)\right. \\
& \left.+\left(r_{\mathrm{c}}+2 d_{\mathrm{s}}-\sigma\right)\left(22 d_{\mathrm{s}}^{2}-5 r_{\mathrm{c}} d_{\mathrm{s}}-7 d_{\mathrm{s}} \sigma-8 r_{\mathrm{c}}^{2}+r_{\mathrm{c}} \sigma+\sigma^{2}\right)\right\}
\end{aligned}
$$


The contact value of the pair distribution function of hard spheres, $g_{\mathrm{hs}}\left(\sigma, n_{i}(\mathbf{r})\right)$, is calculated by using the equation given by $\mathrm{Yu}$ and $\mathrm{Wu}[56]$.

The attractive interactions between all spherical species are described in the framework of the mean field approximation. Assuming that interactions of fluid molecules with all the segments of chains 1 and 2 are identical, we have,

$$
\begin{aligned}
F_{\text {att }} & =\frac{1}{2} \int \mathrm{d} \mathbf{r}_{1} \mathrm{~d} \mathbf{r}_{2} \rho\left(z_{1}\right) \rho\left(z_{2}\right) u_{\mathrm{att}, \mathrm{ff}}\left(r_{12}\right)+\int \mathrm{d} \mathbf{r}_{1} \mathrm{~d} \mathbf{r}_{2} \rho_{\mathrm{s}}^{(1)}\left(z_{1}\right) \rho\left(z_{2}\right) u_{\mathrm{att}, \mathrm{fc}}\left(r_{12}\right) \\
& +\int \mathrm{d} \mathbf{r}_{1} \mathrm{~d} \mathbf{r}_{2} \rho_{\mathrm{s}}^{(2)}\left(z_{1}\right) \rho\left(z_{2}\right) u_{\mathrm{att}, \mathrm{fc}}\left(r_{12}\right)
\end{aligned}
$$

where $u_{\text {att,ff }}(r)$ and $u_{\text {att,fc }}(r)$ are the attractive parts of the fluid-fluid and fluid-segment potentials, respectively.

\section{B. Density profile equations}

Density profile equations are obtained from equation $(15)$. For fluid molecules, we obtain

$$
\rho(z)=\exp \left[\frac{\mu-\lambda(z)}{k T}\right]
$$

where $\lambda(z)=\delta F_{\mathrm{ex}} / \delta \rho(z)+v(z)$. However, the segment density profiles are evaluated taking into account the constraint imposed by equation (14) of the present work. We have

$$
\begin{aligned}
\rho_{\mathrm{s} j}^{(I)}(z) & =R_{\mathrm{c} I} \exp \left[-\lambda_{j}^{(\mathrm{c} I)}(z) / k T\right] G_{j}^{(\mathrm{L} I)}(z) G_{M+1-j}^{(\mathrm{R} I)}(z) \\
& \times\left\{\int_{-H / 2}^{H / 2} \mathrm{~d} z \exp \left[-\lambda_{j}^{(\mathrm{c} I)}(z) / k T\right] G_{j}^{(\mathrm{L} I)}(z) G_{M+1-j}^{(\mathrm{R} I)}(z)\right\}^{-1},
\end{aligned}
$$

where $\lambda_{j}^{(\mathrm{c} I)}(z)=\delta F_{\mathrm{ex}} / \delta \rho_{\mathrm{s}}^{(I)}(z)+v_{\mathrm{s} j}^{(I)}(z)$ and where the functions $G_{j}^{(P I)}(z), P=\mathrm{L}, \mathrm{R}$ are determined from the recurrence relations [54],

$$
G_{j}^{(\mathrm{LI})}(z)=\int \mathrm{d} z^{\prime} \exp \left[-\lambda_{j-1}^{(\mathrm{c} I)}(z) / k T\right] \frac{\theta\left(\sigma_{\mathrm{c}}-\left|z-z^{\prime}\right|\right)}{2 \sigma_{\mathrm{c}}} G_{j-1}^{(\mathrm{L} I)}\left(z^{\prime}\right),
$$

and

$$
G_{j}^{(\mathrm{R} I)}(z)=\int \mathrm{d} z^{\prime} \exp \left[-\lambda_{M-j+2}^{(\mathrm{c} I)}(z) / k T\right] \frac{\theta\left(\sigma_{\mathrm{c}}-\left|z-z^{\prime}\right|\right)}{2 \sigma_{\mathrm{c}}} G_{j-1}^{(\mathrm{R} I)}\left(z^{\prime}\right),
$$

for $i=2,3, \ldots, M$ and with $G_{1}^{(\mathrm{L} I)}(z)=G_{1}^{(\mathrm{R} I)}(z) \equiv 1$.

\section{References}

1. Omelyan I.P., Mryglod I.M., Tokarchuk M.V., Condens. Matter Phys., 2005, 8, 25, doi $10.5488 /$ CMP.8.1.25

2. Patsahan O., Mryglod I., Condens. Matter Phys., 2012, 15, 24001, doi 10.5488/CMP.15.24001.

3. Mryglod I.M., Condens. Matter Phys., 1998, 1, 753, doi:10.5488/CMP.1.4.753.

4. Jonas A.M., Hu Z., Glinel K., Huck W.T.S., Nano Lett., 2008, 8, 3819, doi 10.1021/nl802152q

5. Jonas A.M., Hu Z., Glinel K., Huck W.T.S., Macromolecules, 2008, 41, 6859, doi $10.1021 / \mathrm{ma} 801584 \mathrm{k}$

6. Conrad J.C., Robertson M.L., Curr. Opin. Solid State Mater. Sci., 2019, 23, 1, doi 10.1016/j.cossms.2018.09.004

7. Li B., Yu B., Ye Q., Zhou F., Acc. Chem. Res., 2015, 48, 229, doi 10.1021/ar500323p

8. Minko S., J. Macromol. Sci., Polym. Rev., 2006, 46, 397, doi 10.1080/15583720600945402

9. Uhlmann P., Ionov L., Houbenov N., Nitschke M., Grundke K., Motornov M., Minko S., Stamm M., Prog. Org. Coat., 2006, 55, 168, doi $10.1016 /$ j.porgcoat.2005.09.014

10. Brittain W.J., Minko S., J. Polym. Sci., Part A: Polym. Chem., 2007, 45, 3505, doi $10.1002 /$ pola.22180. 
11. Kuroki H., Gruzd A., Tokarev I., Patsahan T., Ilnytskyi J., Hinrichs K., Minko S., ACS Appl. Mater. Interfaces, 2019, 11, 18268, doi $10.1021 /$ acsami.9b06679

12. Constable A.N., Brittain W.J., Colloids Surf., A, 2007, 308, 123, doi $10.1016 /$ j.colsurfa.2007.05.059

13. Constable A.N., Brittain W.J., Colloids Surf., A, 2011, 380, 128, doi 10.1016/j.colsurfa.2011.02.030

14. Ethier J.G., Hall L.M., Macromolecules, 2018, 51, 9878, doi 10.1021/acs.macromol.8b01373

15. Malfreyt P., Tildesley D.J., Langmuir, 2000, 16, 4732, doi $10.1021 /$ la991396z

16. Dimitrov D., Milchev A., Binder K., J. Chem. Phys., 2007, 127, 084905, doi:10.1063/1.2768525

17. Dimitrov D., Milchev A., Binder K., Macromol. Symp., 2007, 252, 47, doi 10.1002/masy.200750605.

18. Dimitrov D., Milchev A., Binder K., Heermann D.W., Macromol. Theory Simul., 2006, 15, 573, doi $10.1002 /$ mats.200600029

19. Goicochea A.G., Alarcón F., J. Chem. Phys., 2011, 134, 014703, doi 10.1063/1.3517869

20. Grest G.S., Murat M., Macromolecules, 1993, 26, 3108, doi:10.1021/ma00064a019

21. Szleifer I., Carignano M.A., In: Advances in Chemical Physics: Polymeric Systems, Vol. 94, Prigogine I., Rice S.A. (Eds.), Wiley \& Sons, 2007, 165-260.

22. Ilnytskyi J., Sokolowski S., Patsahan T., Condens. Matter Phys., 2013, 16, 13606, doi 10.5488/CMP.16.13606

23. Ilnytskyi J.M., Patsahan T., Sokołowski S., J. Chem. Phys., 2011, 134, 204903, doi $10.1063 / 1.3592562$

24. Sokołowski S., Ilnytskyi J., Pizio O., Condens. Matter Phys., 2014, 17, 12601, doi 10.5488/CMP.17.12601.

25. Milner S.T., Witten T.A., Cates M.E., Europhys. Lett., 1988, 5, 413, doi 10.1209/0295-5075/5/5/006

26. Zhulina E.B., Singh C., Balazs A.C., Macromolecules, 1996, 29, 6338, doi $10.1021 / \mathrm{ma} 960498 \mathrm{i}$

27. Zhulina E.B., Leermakers F., Borisov O.V., Sci. Tech. J. Inf. Technol. Mech. Opt., 2015, 15, 493, doi $10.17586 / 2226-1494-2015-15-3-493-499$

28. Zhulina E.B., Leermaker F.A.M., Borisov O.V., Langmuir, 2015, 31, 6514, doi:10.1021/acs.langmuir.5b00947

29. Lebedeva I.O., Zhulina E.B., Borisov O.V., J. Chem. Phys., 2017, 146, 214901, doi 10.1063/1.4984101.

30. Patrykiejew A., Sokolowski S., Pizio O., In: Surface and Interface Science, Vol. 6, Solid-Gas Interfaces II, Wandelt K. (Ed.), Wiley, Berlin, 2016, 883-1253.

31. Jain S., Dominik A., Chapman W.G., J. Chem. Phys., 2007, 127, 244904, doi $10.1063 / 1.2806932$

32. Jain S., Jog P., Weinhold J., Srivastava R., Chapman W.G., J. Chem. Phys., 2008, 128, 154910, doi $10.1063 / 1.2902976$

33. Gong K., Chapman W.G., J. Chem. Phys., 2011, 135, 214901, doi:10.1063/1.3657830

34. Gong K., Marshall B.D., Chapman W.G., J. Chem. Phys., 2012, 137, 154904, doi $10.1063 / 1.4757860$.

35. Gong K., Marshall B.D., Chapman W.G., J. Chem. Phys., 2013, 139, 094904, doi 10.1063/1.4819957.

36. Yu Y.-X., Wu J., J. Chem. Phys., 2002, 117, 2368, doi $10.1063 / 1.1491240$

37. Yu Y.-X., Wu J., J. Chem. Phys., 2002, 117, 10156, doi $10.1063 / 1.1520530$

38. Trejos V.M., Pizio O., Sokolowski S., Fluid Phase Equilib., 2018, 473, 145, doi:10.1016/j.fluid.2018.06.005

39. Trejos V.M., Sokołowski S., Pizio O., J. Chem. Phys., 2018, 149, 134701, doi 10.1063/1.5047018

40. Trejos V.M., Pizio O., Sokołowski S., J. Chem. Phys., 2018, 149, 234703, doi $10.1063 / 1.5066552$

41. Trejos V.M., Sokołowski S., Pizio O., Mol. Phys., 2018, 118, 1615647, doi $10.1080 / 00268976.2019 .1615647$

42. Trejos V.M., Pizio O., Sokołowski S., J. Chem. Phys., 2019, 151, 064704, doi 10.1063/1.5116128

43. Clark G.N.I., Haslam A.J., Galindo A., Jackson G., Mol. Phys., 2006, 104, 3561, doi $10.1080 / 00268970601081475$.

44. Gil-Villegas A., Galindo A., Whitehead P.J., Mills S.J., Jackson G., Burgess A.N., J. Chem. Phys., 1997, 106, 4168, doi:10.1063/1.473101.

45. Ulberg D.E., Gubbins K.E., Mol. Simul., 1994, 13, 205, doi:10.1080/08927029408021984

46. Ulberg D.E., Gubbins K.E., Mol. Phys., 1995, 84, 1139, doi:10.1080/00268979500100801

47. Srivastava R., Docherty H., Singh J.K., Cummings P.T., J. Phys. Chem. C, 2011, 115, 12448 , doi $10.1021 / \mathrm{jp} 2003563$

48. Kolafa J., Nezbeda I., Mol. Phys., 1987, 61, 161, doi 10.1080/00268978700101051

49. Jackson G., Chapman W.G., Gubbins K.E., Mol. Phys., 1988, 65, 1, doi $10.1080 / 00268978800100821$

50. Chapman W.G., Jackson G., Gubbins K.E., Mol. Phys., 1988, 65, 1057, doi:10.1080/00268978800101601

51. Steele W.A., The Interaction of Gases with Solid Surfaces, Pergamon Press, Oxford, 1974.

52. Steele W.A., Surf. Sci., 1973, 36, 317, doi 10.1016/0039-6028(73)90264-1

53. Cao D., Wu J., Langmuir, 2006, 22, 2712, doi $10.1021 / 1 a 0527588$.

54. Pizio O., Borówko M., Rżysko W., Staszewski T., Sokołowski S., J. Chem. Phys., 2008, 128, 044702, doi $10.1063 / 1.2829247$

55. Pizio O., Sokołowski S., Sokołowska Z., J. Chem. Phys., 2011, 134, 214702, doi 10.1063/1.3597773

56. Yu Y.-X., Wu J., J. Chem. Phys., 2002, 116, 7094, doi $10.1063 / 1.1463435$

57. Roth R., Evans R., Lang A., Kahl G., J. Phys.: Condens. Matter, 2002, 14, 12063 , doi $10.1088 / 0953-8984 / 14 / 46 / 313$ 
58. Bryk P., Sokołowski S., Pizio O., J. Chem. Phys., 2006, 125, 024909, doi $10.1063 / 1.2212944$

59. Wertheim M.S., J. Stat. Phys., 1984, 35, 19, doi $10.1007 /$ BF01017362.

60. Wertheim M.S., J. Stat. Phys., 1984, 35, 35, doi 10.1007/BF01017363.

61. Wertheim M.S., J. Stat. Phys., 1986, 42, 459, doi 10.1007/BF01127721.

62. Wertheim M.S., J. Stat. Phys., 1986, 42, 477, doi 10.1007/BF01127722.

63. Ahrens H., Förster S., Helm C.A., Kumar N.A., Naji A., Netz R.R., Seidel C., J. Phys. Chem. B, 2004, 108, 16870, doi:10.1021/jp049553c.

64. Pizio O., In: Computational Methods in Surface and Colloid Science, Borówko M. (Ed.), Marcel Dekker, New York, 2000, 293-346.

65. Pizio O., Sokolowski S., J. Phys. Stud., 1998, 2, 296.

66. Borówko M., Rżysko W., Sokołowski S., Staszewski T., J. Phys. Chem. B, 2009, 113, 4763, doi: $10.1021 / \mathrm{jp} 811143 \mathrm{n}$

67. Bucior K., Patrykiejew A., Pizio O., Sokołowski S., J. Colloid Interface Sci., 2003, 259, 209, doi $10.1016 /$ S0021-9797(02)00203-5

\title{
Опис адсорбції води у щілиноподібних наноканалах 3 прищепленими молекулярними щітками. Теорія функціоналу густини
}

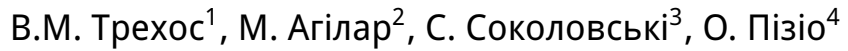 \\ ${ }^{1}$ Інститут фундаментальних наук та інженерії, Автономний університет штату Гідальго, Гідальго, Мексика \\ 2 Інститут хімії, Національний автономний університет м. Мехіко, Мехіко, Мексика \\ 3 Відділ моделювання фізико-хімічних процесів, університет Марії Склодовської-Кюрі, \\ Люблін 20-031, Польща \\ ${ }^{4}$ Інститут хімії, Національний автономний університет м. Мехіко, Мехіко, Мексика
}

Ми дослідили модель адсорбції води у щілиноподібні наноканали з звома стінками, хімічно модифікованими за допомогою прищеплених полімерних шарів, що формують щітки. В якості теоретичних інструментів використано один з варіантів методу функціоналу густини. Модель водоподібного плину, запозичена з роботи Кларка та ін. [Mol. Phys., 2006, 104, 3561] адекватно відтворює співіснування пара-рідина в об'ємі. Полімерний шар складається з ланцюжкових молекул в рамках моделі намиста (pearl-necklace model). Кожна ланцюжкова молекула хімічно зв'язана зі стінками пор одним завершальним сегментом. Основною метою даного дослідження $\epsilon$ вивчення залежності висоти полімерного шару від густини прищеплення та мікроскопічної структури інтерфейсу між адсорбованим плином і щітками. Детально досліджено термічний відгук цих властивостей на адсорбцію. Отримані результати є важливими для розуміння стягування і набрякання молекулярних щіток у наноканалах.

Ключові слова: асоційовані плини, теорія функціоналу густини, адсорбція, молекулярні щітки, модель води 


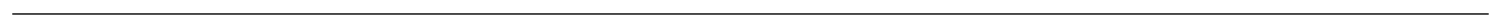

